\title{
A combination of high concentrations of serum triglyceride and non-high-density-lipoprotein-cholesterol is a risk factor for cardiovascular disease in subjects with abnormal glucose metabolism-The Hoorn Study
}

\author{
G. Bos ${ }^{1}$, J. M. Dekker ${ }^{1}$, G. Nijpels ${ }^{1}$, F. de $\operatorname{Vegt}^{2}$, M. Diamant ${ }^{3}$, C. D. A. Stehouwer ${ }^{1,4}$, L. M. Bouter ${ }^{1}$, \\ R. J. Heine ${ }^{1,3}$ \\ ${ }^{1}$ Institute for Research in Extramural Medicine, VU University Medical Center, Amsterdam, The Netherlands \\ 2 Department of Epidemiology and Biostatistics, University Medical Centre St Radboud Nijmegen \\ ${ }^{3}$ Department of Endocrinology/Diabetes Center, VU University Medical Center, Amsterdam \\ ${ }^{4}$ Institute for Cardiovascular Research, VU University Medical Center, Amsterdam, The Netherlands
}

\section{Abstract}

Aims/hypothesis. Type 2 diabetes is not only associated with hyperglycaemia, but also with disorders of lipid metabolism. The aim of this study was to investigate the association of triglyceride and non-HDL-cholesterol concentrations with cardiovascular disease in subjects with normal and abnormal glucose metabolism.

Methods. Subjects were 869 men and 948 women aged 50 to 75 who participated in the Hoorn Study, a population-based cohort study that started in 1989 . Glucose metabolism was determined by a 75 g OGTT. High fasting triglyceride and non-HDL-cholesterol concentrations were defined as above the median of the study population.

Results. After 10 years of follow-up, the age- and sexadjusted hazard ratios for cardiovascular disease were 1.35 (1.11-1.64) and 1.71 (1.40-2.08) for high triglycerides and high non-HDL-cholesterol, respectively, after mutual adjustment. After stratification for glucose metabolism status, the hazard ratios for cardiovascular disease for non-HDL-cholesterol were $1.70(1.31-2.21)$ in normal glucose metabolism and 1.56 (1.12-2.18) in abnormal glucose metabolism. Triglycerides were not a risk factor in subjects with normal glucose metabolism, with a hazard ratio of 0.94 (0.73-1.22), but in subjects with abnormal glucose metabolism, the hazard ratio for cardiovascular disease was 1.54 (1.07-2.22). In subjects with abnormal glucose metabolism, the hazard ratio for the combined presence of high triglycerides and non-HDLcholesterol was 2.12 (1.35-3.34).

Conclusion. Our data suggest that in people with abnormal glucose metabolism, but not in those with normal glucose metabolism, high triglyceride concentration could be associated with the risk of cardiovascular disease, particularly in people with high nonHDL-cholesterol. [Diabetologia (2003) 46:910-916]

Keywords Type 2 diabetes, triglycerides, non-HDLcholesterol, cardiovascular disease, insulin-resistance.
Type 2 (non-insulin-dependent) diabetes is associated with a two- to four-fold increased risk of cardiovascular disease [1, 2, 3]. Recently, the United Kingdom

Received: 30 December 2002 / Revised: 28 February 2003 Published online: 18 June 2003

C Springer-Verlag 2003

Corresponding author: G. Bos, Institute for Research in Extramural Medicine, VU University Medical Center, Van der Boechorststraat 7, 1081 BT Amsterdam, The Netherlands E-mail: g.bos.emgo@med.vu.nl

Abbreviations: ICD, International Classification of Diseases; IDL, intermediate-density-lipoprotein; TRL, triglyceride-rich lipoproteins.
Prospective Diabetes Study showed that an increased concentration of low-density-lipoprotein (LDL)-cholesterol and decreased concentration of high-densitylipoprotein (HDL)-cholesterol, systolic blood pressure, hyperglycaemia, and smoking are the strongest risk factors for cardiovascular mortality and morbidity in patients with Type 2 diabetes [4].

A growing body of evidence now supports the role of triglyceride concentration as an important risk factor for cardiovascular disease [5], but different results have been found in specific subgroups, for instance in subjects with Type 2 diabetes and in obese, insulin-resistant subjects [6, 7]. High concentrations of triglycerides are typically observed in people with Type 2 dia- 
betes. In contrast, total cholesterol and LDL-cholesterol concentrations have not been found to differ between people with and without Type 2 diabetes [8]. Very low-density-lipoprotein (VLDL) and intermediate-density-lipoprotein (IDL) remnants are triglyceride-rich lipoproteins (TRL) and have been shown to possess atherogenic properties. It has been proposed that non-HDL-cholesterol, which includes all cholesterol in atherogenic lipoprotein particles, such as LDL, Lp(a), VLDL and IDL, might be a stronger risk factor for cardiovascular disease than LDL-cholesterol concentration. In the Quebec Cardiovascular Study, high apoB, representing LDL, VLDL and IDL, was associated with a more than two-fold risk of ischaemic heart disease [9]. High non-HDL-cholesterol could be particularly relevant in people with Type 2 diabetes and hypertriglyceridaemia $[10,11,12,13,14]$. It has been proposed that the triglycerides in the non-HDLparticles could further enhance the atherogenic potential of these particles [15].

The objectives of this study were to investigate the association of triglyceride concentrations with subsequent cardiovascular disease, in a population-based prospective cohort study (the Hoorn Study) of men and women aged 50 to 75 years [16], and to examine the association of non-HDL-cholesterol concentration with cardiovascular disease in subjects with low or high triglyceride concentrations. Finally, to determine whether these associations differ between subjects with normal and abnormal glucose metabolism.

\section{Materials and methods}

Study cohort and methods. All subjects were participants in the Hoorn Study, a prospective population-based cohort study of glucose metabolism and diabetes complications, as described [16]. Briefly, in 1989, a random sample of 50- to 75-year-old individuals was taken from the population register of the town of Hoorn in the Netherlands. Of the 3552 individuals invited to take part in the study, 2540 participated $(71.5 \%)$, of whom 56 non-Caucasians were excluded, resulting in 2484 participants at baseline. A $75 \mathrm{~g}$ OGTT was carried out in all participants, except in Type 2 diabetic patients using oral blood glucose lowering agents or insulin. Four glucose metabolism categories were defined according to the WHO-99 criteria [17]. Subjects with impaired glucose metabolism (impaired fasting glucose or impaired glucose tolerance), or known or newly diagnosed Type 2 diabetes were grouped together in the 'abnormal glucose metabolism' group and compared to subjects with normal glucose metabolism. Fasting plasma glucose concentration and 2-h post-load plasma glucose concentration were determined with a glucose dehydrogenase method (Merck, Darmstadt, Germany). After precipitation of the LDL and VLDL from fasting blood samples, triglycerides, total cholesterol and HDL-cholesterol were measured by enzymatic techniques (Boehringer-Mannheim, Mannheim, Germany). Non-HDLcholesterol concentration was defined as the difference between total cholesterol and HDL-cholesterol concentration. Systolic and diastolic blood pressure were determined at the right upper-arm, after 5 min of rest in seated subjects, using a random-zero sphygmomanometer (Hawksley-Gelman, Lancing,
UK). The average of duplicate measurements was used. On their visit to the research centre, participants were asked to bring their medication; name and dosages of medicaments were recorded and coded. Anthropometric data were obtained from all subjects, wearing light clothes only. BMI and waisthip-ratio were calculated by dividing weight by height square, and waist circumference by hip circumference, respectively. Cardiovascular history and lifestyle habits, including smoking and alcohol intake were assessed by questionnaire. We excluded people with missing data on glucose or lipids $(n=18)$, individuals using lipid-lowering drugs $(n=34)$ at baseline, individuals with missing morbidity data $(n=612)$, and individuals with missing cause-specific mortality data $(n=32)$. Consequently, the study population consisted of 1817 subjects, 869 men and 948 women. The Ethical Review Committee of the VU University Medical Centre approved the Hoorn Study. Informed consent was obtained from all participants.

Follow-up and definition of cardiovascular disease. In 1995 the population register of Hoorn was checked for the vital status of all participants, and since then a prospective registration was continued. In this study, follow-up data until 1st January 2000 were used. Information about non-fatal cardiovascular disease was obtained by searching medical records from the local hospital of the town of Hoorn; causes of death were extracted from the medical records of the general practitioner and the local hospital. Both fatal and non-fatal cardiovascular disease were coded according to the International Classification of Diseases (ICD-9) [18]. Cardiovascular disease was defined as first new cardiovascular event. Non-fatal cardiovascular disease was defined as coronary heart disease (defined by prolonged typical chest pain, ECG changes and enzymes), congestive heart failure, transient ischaemic attack or stroke, or peripheral atherosclerotic disease. Fatal cardiovascular disease was defined as ICD codes 390-459 ('Diseases of the circulatory system') or 798 ('Sudden death, cause unknown'). A computer program was used to classify cardiovascular disease, and in case of doubt a specialist in internal medicine or a cardiologist decided about the events. Seventeen subjects $(0.7 \%)$ were lost to follow-up. We did not receive information about morbidity from 612 individuals $(24.6 \%)$, because they did not give permission to access hospital files or had moved out of the town of Hoorn. Follow-up duration was calculated from the date of baseline examinations to date of death, date of first new cardiovascular event after baseline, date of loss to follow-up, or to 1st January 2000 in event-free subjects.

Statistical analysis. Firstly, we determined the contribution of high concentrations of triglyceride and non-HDL-cholesterol to the development of cardiovascular disease. Furthermore, we assessed the influence of the combined presence of high or low concentrations of triglyceride and non-HDL-cholesterol. For this analysis, subjects were categorized into four groups $(2 \times 2)$ according to the median values of triglyceride and non-HDLcholesterol, i.e. $1.4 \mathrm{mmol} / \mathrm{l}$ for men and $1.3 \mathrm{mmol} / \mathrm{l}$ for women, and $5.2 \mathrm{mmol} / \mathrm{l}$ for men and $5.3 \mathrm{mmol} / \mathrm{l}$ for women, respectively. We chose to create groups on the basis of medians in order to 'fill' the groups with low/high combinations of triglycerides and non-HDL-cholesterol and vice versa, which are less common than low/low or high/high combinations. Baseline characteristics are shown as means with standard deviations, but for triglyceride concentration (skewed data) the median and interquartile ranges are shown. Group differences in continuous variables were examined using ANOVA; for dichotomous variables, logistic regression was used. Incidence densities were calculated on the basis of on person-years of follow-up. With 10 years of follow-up and 408 cases of cardiovascular disease, 
Table 1. Baseline characteristics of the study population according to triglyceride (TG) and non-HDL-cholesterol levels

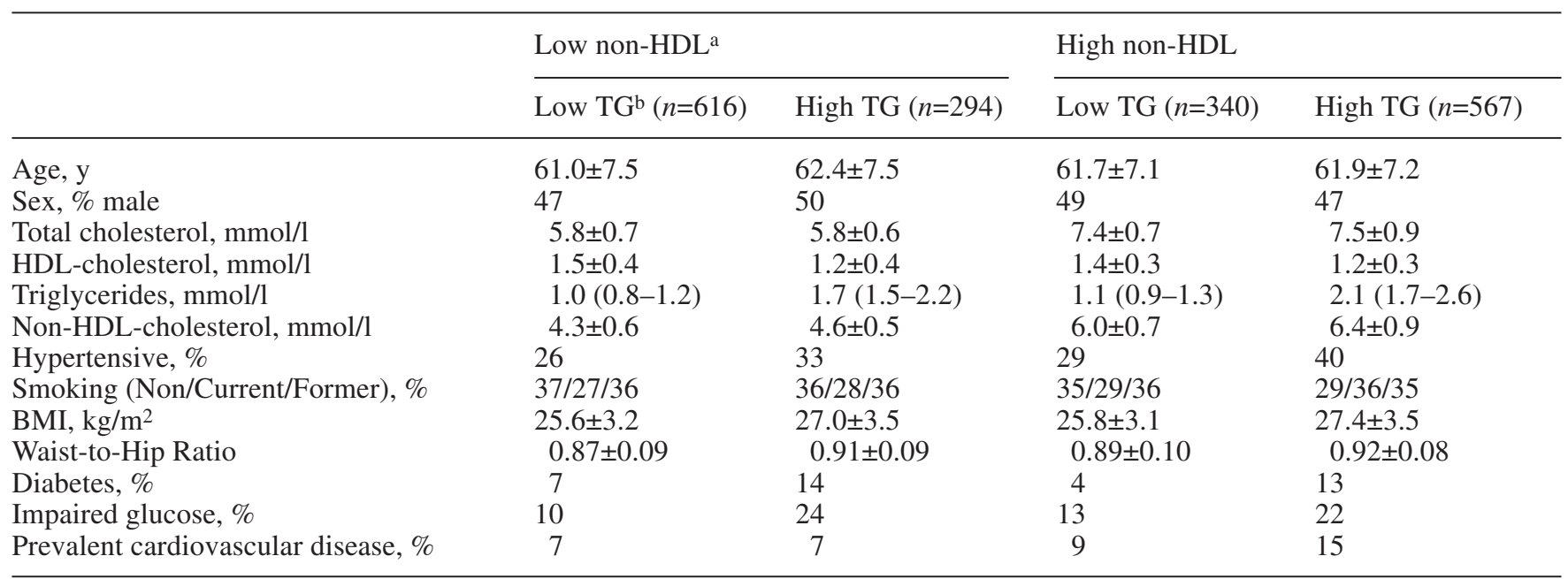

a Cut-off points for non-HDL-cholesterol are $5.2 \mathrm{mmol} / \mathrm{l}$ in men and $5.3 \mathrm{mmol} / \mathrm{l}$ in women (medians)

b Cut-off points for triglycerides are $1.4 \mathrm{mmol} / \mathrm{l}$ in men and $1.3 \mathrm{mmol} / \mathrm{l}$ in women (medians)

hazard ratios and $95 \% \mathrm{CI}$ of baseline triglyceride and nonHDL-cholesterol concentrations for cardiovascular disease were obtained from multivariate Cox proportional hazard models. We first adjusted for age and sex, then for other potentially confounding variables (i.e. waist-to-hip ratio, hypertension, prevalent cardiovascular disease, smoking and alcohol consumption). Since triglycerides are strongly associated with factors of the insulin-resistance syndrome, a multivariate model was conducted only in the final stratified analyses, because of risk of over adjustment. Furthermore, we tested for eventual interaction between triglyceride, non-HDL-cholesterol and glucose metabolism status by adding a product term to the survival models (triglyceride $\times$ non-HDL-cholesterol, or triglyceride $\times$ non-HDL-cholesterol $\times(\mathrm{ab})$ normal glucose metabolism). A $p$ value of less than 0.05 was considered statistically significant. Statistical analyses were done using SPSS for Windows version 10.1.4.

\section{Results}

The baseline characteristics of subgroups classified on the basis of triglyceride and non-HDL-cholesterol concentrations are shown (Table 1). Among the subjects with high triglyceride concentrations, there were more Type 2 diabetic patients and subjects with impaired glucose metabolism. High concentration of non-HDL-cholesterol was associated with prevalent cardiovascular disease, in both triglyceride categories.

The hazard ratios of cardiovascular disease were 1.19 and 1.63 for subjects with high triglyceride and high non-HDL-cholesterol concentrations, respectively, after mutual adjustment. The hazard ratio for subjects with both high triglyceride and high nonHDL-cholesterol concentrations was 1.92 (1.50-2.47) (Table 2).

Subsequently we studied the eventual differences in the strength of these associations between people
Table 2. Hazard ratios (95\% CI) of cardiovascular disease (CVD) for triglycerides (TG) and non-HDL-cholesterol

\begin{tabular}{|c|c|c|c|}
\hline & $n$ & Cases & CVD \\
\hline \multicolumn{4}{|l|}{ Linear } \\
\hline \multicolumn{4}{|l|}{ Age- and sex-adjusted } \\
\hline TG (log-transformed) & 1817 & 408 & $1.82(1.49-2.24)$ \\
\hline Non-HDL & 1817 & 408 & $1.34(1.24-1.45)$ \\
\hline \multicolumn{4}{|l|}{ Mutually adjusted ${ }^{a}$} \\
\hline TG (log-transformed) & 1817 & 408 & $1.39(1.10-1.76)^{b}$ \\
\hline Non-HDL & 1817 & 408 & $1.26(1.15-1.38)^{\mathrm{e}}$ \\
\hline \multicolumn{4}{|l|}{ Dichotomised } \\
\hline \multicolumn{4}{|l|}{ Age- and sex-adjusted } \\
\hline High $\mathrm{TG}^{\mathrm{c}}$ & 861 & 223 & $1.35(1.11-1.64)$ \\
\hline High non-HDL ${ }^{d}$ & 907 & 248 & $1.71(1.40-2.08)$ \\
\hline \multicolumn{4}{|l|}{ Mutually adjusted ${ }^{a}$} \\
\hline High TG & 861 & 223 & $1.19(0.98-1.46)^{\mathrm{e}}$ \\
\hline High non-HDL & 907 & 248 & $1.63(1.33-2.01)^{\mathrm{e}}$ \\
\hline \multicolumn{4}{|l|}{ Combined categories ${ }^{a}$} \\
\hline Low non-HDL, low TG & 616 & 100 & 1 \\
\hline Low non-HDL, high TG & 294 & 60 & $1.10(0.80-1.52)$ \\
\hline High non-HDL, low TG & 340 & 85 & $1.53(1.15-2.04)$ \\
\hline High non-HDL, high TG & 567 & 163 & $1.92(1.50-2.47)$ \\
\hline
\end{tabular}

\footnotetext{
a Age- and sex-adjusted

$\mathrm{b}$ Interaction between triglyceride*non-HDL-cholesterol $\mathrm{p}=0.65$

${ }^{c}$ Cut-off points for triglycerides are $1.4 \mathrm{mmol} / \mathrm{l}$ in men and $1.3 \mathrm{mmol} / \mathrm{l}$ in women (medians)

${ }^{\mathrm{d}}$ Cut-off points for non-HDL-cholesterol are $5.2 \mathrm{mmol} / \mathrm{l}$ in men and $5.3 \mathrm{mmol} / \mathrm{l}$ in women (medians)

${ }^{\mathrm{e}}$ Interaction between triglyceride*non-HDL-cholesterol $p=0.53$
}

with normal and abnormal glucose metabolism. In abnormal glucose metabolism, but not in normal glucose metabolism, the combined presence of high concentration of triglyceride and non-HDL-cholesterol 
Table 3. Hazard ratios (95\% CI) of cardiovascular disease (CVD) for medians of triglycerides (TG) and non-HDL-cholesterol, stratified for glucose metabolism

\begin{tabular}{|c|c|c|c|c|c|c|}
\hline & \multicolumn{3}{|c|}{ Normal glucose metabolism } & \multicolumn{3}{|c|}{ Abnormal glucose metabolism } \\
\hline & $n$ & Cases & CVD & $n$ & Cases & CVD \\
\hline \multicolumn{7}{|l|}{ Dichotomised } \\
\hline \multicolumn{7}{|l|}{ Age- and sex-adjusted } \\
\hline High $\mathrm{TG}^{\mathrm{a}}$ & 547 & 112 & $1.10(0.86-1.41)$ & 314 & 111 & $1.67(1.16-2.39)$ \\
\hline High non-HDL b & 647 & 153 & $1.67(1.30-2.15)$ & 260 & 95 & $1.67(1.20-2.33)$ \\
\hline \multicolumn{7}{|l|}{ Mutually adjusted ${ }^{\mathrm{c}}$} \\
\hline High TG & 547 & 112 & $0.94(0.73-1.22)^{d}$ & 314 & 111 & $1.54(1.07-2.22)^{\mathrm{d}}$ \\
\hline High non-HDL & 647 & 153 & $1.70(1.31-2.21)^{\mathrm{d}}$ & 260 & 95 & $1.56(1.12-2.18)^{\mathrm{d}}$ \\
\hline \multicolumn{7}{|l|}{ Multivariate model $\mathrm{e}$} \\
\hline High TG & 529 & 105 & $0.70(0.53-0.92)$ & 303 & 106 & $1.48(1.00-2.20)$ \\
\hline High non-HDL & 629 & 144 & $1.54(1.17-2.03)$ & 253 & 90 & $1.20(0.84-1.71)$ \\
\hline \multicolumn{7}{|l|}{ Combined categories } \\
\hline \multicolumn{7}{|l|}{ Age- and sex-adjusted } \\
\hline Low non-HDL, low TG & 509 & 75 & 1 & 107 & 25 & 1 \\
\hline Low non-HDL, high TG & 181 & 28 & $0.96(0.62-1.48)$ & 113 & 32 & $1.03(0.55-1.93)$ \\
\hline High non-HDL, low TG & 281 & 69 & $1.71(1.23-2.37)$ & 59 & 16 & $1.13(0.67-1.91)$ \\
\hline High non-HDL, high TG & 366 & 84 & $1.60(1.17-2.19)$ & 201 & 79 & $2.12(1.35-3.34)$ \\
\hline \multicolumn{7}{|l|}{ Multivariate model e } \\
\hline Low non-HDL, low TG & 497 & 72 & 1 & 105 & 24 & 1 \\
\hline Low non-HDL, high TG & 173 & 25 & $0.69(0.44-1.10)$ & 109 & 32 & $1.38(0.79-2.41)$ \\
\hline High non-HDL, low TG & 273 & 64 & $1.54(1.09-2.16)$ & 59 & 16 & $1.09(0.57-2.07)$ \\
\hline High non-HDL, high TG & 356 & 80 & $1.07(0.77-1.50)$ & 194 & 74 & $1.73(1.06-2.81)$ \\
\hline
\end{tabular}

a Cut-off points for triglycerides are $1.4 \mathrm{mmol} / \mathrm{l}$ in men and $1.3 \mathrm{mmol} / \mathrm{l}$ in women (medians)

${ }^{\mathrm{b}}$ Cut-off points for non-HDL-cholesterol are $5.2 \mathrm{mmol} / \mathrm{l}$ in men and $5.3 \mathrm{mmol} / \mathrm{l}$ in women (medians)

c Age- and sex-adjusted

was associated with a two-fold higher incident density for cardiovascular disease (Fig. 1). The figure suggests an interaction (effect-modification) between triglyceride concentration and non-HDL-cholesterol concentration and glucose metabolism, such that in subjects with abnormal glucose metabolism with a high non-HDL-cholesterol concentration in particular, high triglyceride was associated with a higher risk of cardiovascular disease. However, a test for interaction in the Cox model was not significant $(p=0.14)$. In normal and abnormal glucose metabolism, non-HDLcholesterol concentration was a risk factor for cardiovascular disease. Subjects with high non-HDL-cholesterol concentration had a $70 \%$ higher cardiovascular risk than subjects with low non-HDL-cholesterol concentration, independently of triglyceride concentration (Table 3). A high concentration of triglyceride was associated with a $50 \%$ higher risk of cardiovascular disease in subjects with abnormal glucose metabolism, but not in those with normal glucose metabolism. Adjustment for other potential confounders attenuated the observed associations between the triglyceride and non-HDL-cholesterol concentrations and d Interaction between triglyceride*non-HDL-cholesterol*glucose metabolism status $p=0.14$

e Adjusted for age, sex, waist-to-hip ratio, hypertension, prevalent cardiovascular disease, smoking and alcohol consumption

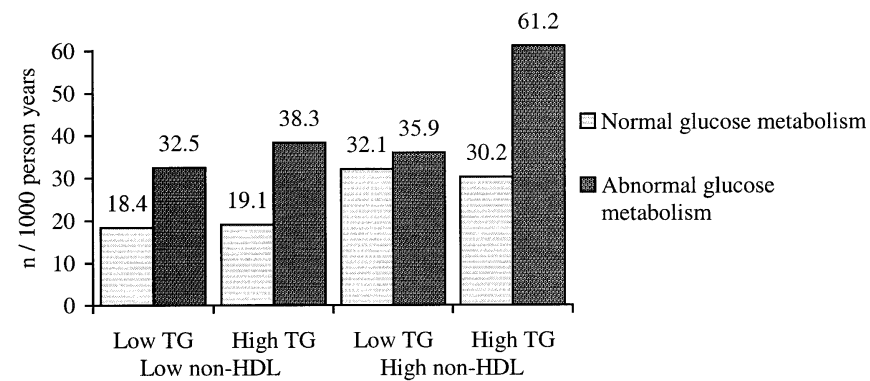

Fig. 1. Cardiovascular disease for combined categories of triglycerides (TG) and non-HDL-cholesterol in subjects with normal $(n=1337)$ and abnormal glucose metabolism $(n=480)$. Bars represent unadjusted incidence densities

cardiovascular disease in either group. The combined presence of high triglycerides and non-HDL-cholesterol in abnormal glucose metabolism was associated with risk of cardiovascular disease, with a hazard ratio of 1.73. Even adjustment for HDL-cholesterol did not materially alter the hazard ratio $[1.68$ (1.01-2.78)] (Fig. 2). 


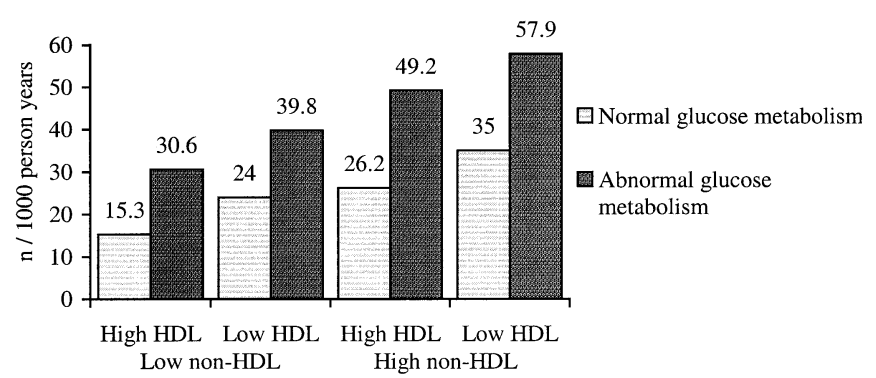

Fig. 2. Cardiovascular disease for combined categories of HDL-cholesterol and non-HDL-cholesterol in subjects with normal $(n=1337)$ and abnormal glucose metabolism $(n=480)$. Bars represent unadjusted incidence densities

\section{Discussion}

In this study, we observed that high triglyceride concentrations did not contribute independently to cardiovascular risk in subjects with normal glucose metabolism, whereas there was a substantially increased risk in subjects with abnormal glucose metabolism.

There is a large body of evidencing large studies that triglyceride concentration is a risk factor for cardiovascular disease for both non-diabetic subjects and diabetic patients $[19,20,21,22,23,24,25]$. A recent meta-analysis of 17 prospective trials with 57,000 subjects also concluded that triglyceride concentration is a risk factor in non-diabetic subjects [5]. We observed that high triglyceride concentrations are a risk factor for cardiovascular disease in subjects with abnormal glucose metabolism, but only in those with high non-HDL-cholesterol. This result supports the inference that the predictive value of triglycerides for cardiovascular risk depends on population and the lipoprotein profile [26].

Many studies have shown that high serum cholesterol concentration, especially LDL-cholesterol concentration, and low HDL-cholesterol concentration are risk factors for cardiovascular disease in the general population [25, 27]. In the Lipid Research Clinics Program Follow-up Study, non-HDL-cholesterol concentration was a stronger risk factor for cardiovascular disease mortality than LDL-cholesterol concentration [10]. More recently, investigators of the Bypass Angioplasty Revascularization Investigation concluded that non-HDL-cholesterol was a predictor of coronary heart disease [28]. This is in line with findings in amongst others the Systolic Hypertension in the Elderly Program, where non-HDL-cholesterol concentration was a risk factor for cardiovascular disease in the elderly population consisting of 4736 men and women with systolic hypertension [29]. Recently, also the Strong Heart Study reported that non-HDL-cholesterol could be useful in predicting cardiovascular risk in patients with diabetes [30]. Since non-HDL-cholesterol includes not only LDL-cholesterol, but also cholesterol in potentially atherogenic TRL, notably rem- nant proteins, it could indeed identify more atherogenic lipoproteins than does LDL-cholesterol concentration alone.

There are several possible explanations for the discrepant contribution of increased triglyceride concentrations to cardiovascular risk between persons with and without abnormal glucose metabolism. High fasting and in particular postprandial concentrations of triglycerides in subjects with abnormal glucose metabolism are due to defective insulin action in the liver and peripheral tissues [31], resulting in impaired suppression of hepatic VLDL production, reduced lipoprotein lipase activity and consequently impaired clearance of triglyceride-rich particles due to competition for the same pathways. Together these events in turn will lead to longer circulation of atherogenic triglyceride-rich particles [32]. High triglyceride concentration, therefore, could be indicative of a more atherogenic composition of the non-HDL-cholesterol particles, for example small dense LDL, especially in people who have abnormal glucose metabolism [33]. It has been suggested to distinguish "good" and "bad" hypertriglyceridaemia on the basis of the association with risk of cardiovascular disease [15]. "Bad" triglycerides were suggested to represent increased concentrations of apoC-III on the apoB-containing lipoproteins, resulting in a diminished ability of lipoprotein lipase to hydrolyse the triglycerides in the particles, leading to accumulation of atherogenic remnants. In contrast, the "good" triglyceride "cluster" constitutes of high or normal HDL-cholesterol concentration and low apoC-III and is not associated with enhanced cardiovascular risk.

The finding that high triglycerides were protective in subjects with normal glucose metabolism in the multivariate model was somewhat unexpected. It could be that this is a finding in our population by chance; it could be due to statistical limitations. None of the other individual risk factors actually caused the relative risk of high triglycerides to be protective. Only when adding them simultaneously, the hazard ratio became 0.70 . This might be caused by overadjustment, which is adjusting for factors that are part of the causal chain, or it might be true. The remaining triglycerides could reflect the "good" triglycerides, rendering LDL large and buoyant, and in HDL-particles [15]. Besides, we expected to find an interaction between triglyceride concentration and non-HDL-cholesterol concentration as cardiovascular risk factors in subjects with abnormal glucose metabolism. However, when testing for interaction, it turned out to be not statistically significant $(p=0.14)$. Despite this, we conducted survival analysis in strata of glucose metabolism for reasons based on the mechanism of hypertriglyceridaemia in subjects with abnormal glucose metabolism. Indeed, we found support for this concept in our data. The fact that the interaction was not statistically significant, possibly due to lack of power. 
In this population study, there is a strong correlation between triglyceride and non-HDL-cholesterol concentrations (Spearman correlation coefficient was 0.48). Since non-HDL-cholesterol, TRL and triglycerides are interrelated, their individual contribution to the pathological processes of cardiovascular disease cannot be readily assessed.

In this 10-year follow-up study we were able to compare the risk of triglyceride concentration and non-HDL-cholesterol concentration with cardiovascular disease between subjects with normal and abnormal glucose metabolism in a well-characterized population-based cohort study. Diabetic patients and subjects with impaired glucose tolerance were taken together in the analyses. Earlier studies have shown that the adverse lipid profile is similar for subjects with impaired glucose metabolism and subjects with newly-diagnosed Type 2 diabetes [34]. Additional analyses, in which people with impaired glucose metabolism and newly-diagnosed diabetic subjects were considered separately, yielded similar results (data not shown). The number of known diabetic subjects $(n=68)$ was too small to be analysed separately. Exclusion of known diabetic subjects did not alter the results (data not shown).

A limitation of our study was that 612 subjects had to be excluded because they did not give permission to access hospital files. However, there were no considerable differences between individuals who were excluded and the rest of the cohort with respect to cardiovascular risk factors at baseline (data not shown). In addition, we analysed the impact of exclusion of prevalent cardiovascular disease. Exclusion of subjects with prevalent cardiovascular disease at baseline did not alter the results in normal nor in abnormal glucose metabolism, and in the overall cohort. All results were similar for fatal and non-fatal cardiovascular disease (data not shown).

The triglyceride concentration was obtained by a single-fasting plasma triglyceride sample. Although there is a considerable intra-individual variation in fasting triglyceride concentration, in a large epidemiological study, it is still possible to categorize the majority of the subjects correctly into broad subgroups with high and low concentrations of triglycerides. It is expected that a (small) proportion of people who in general have a mean high concentration are wrongly classified as low, and vice versa. This would have led to an underestimation of the real relative risk.

In conclusion, in a prospective population-based cohort study, we observed that triglyceride concentration was not a risk factor for cardiovascular disease in subjects with normal glucose metabolism, whereas triglyceride concentration contributed to cardiovascular disease risk in subjects with abnormal glucose metabolism. We found a strong positive association between serum non-HDL-cholesterol concentration and future cardiovascular risk, independent of triglyceride concentration. In subjects with abnormal glucose metabo- lism, the increased risk of triglyceride concentrations above the median of the population and high concentration of non-HDL-cholesterol associated with risk of cardiovascular disease seems to be even more explicit than in subjects with normal glucose metabolism. Monitoring triglyceride concentrations in people with abnormal glucose metabolism could contribute to the cardiovascular risk assessment.

Acknowledgements. We thank the Westfries Gasthuis, the participating general practitioners, and the population register of the town of Hoorn for their co-operation with this study. We also thank R. Last for carefully searching the medical records for evidence of cardiovascular disease. The project was funded by the Dutch Diabetes Research Foundation and the Nederlandse Organisatie voor Wetenschappelijk Onderzoek (NWO), and their support is gratefully acknowledged.

\section{References}

1. Laakso M (1999) Hyperglycemia and cardiovascular disease in type 2 diabetes. Diabetes 48:937-942

2. De Vegt F, Dekker JM, Ruhe HG et al. (1999) Hyperglycaemia is associated with all-cause and cardiovascular mortality in the Hoorn population: the Hoorn Study. Diabetologia 42:926-931

3. Kannel WB, McGee DL (1979) Diabetes and cardiovascular risk factors: the Framingham study. Circulation 59:8-13

4. Turner RC, Millns H, Neil HA et al. (1998) Risk factors for coronary artery disease in non-insulin dependent diabetes mellitus: United Kingdom Prospective Diabetes Study (UKPDS: 23). BMJ 316:823-828

5. Hokanson JE, Austin MA (1996) Plasma triglyceride level is a risk factor for cardiovascular disease independent of high-density lipoprotein cholesterol level: a meta-analysis of population-based prospective studies. J Cardiovasc Risk 3:213-219

6. Assmann G, Schulte H, Funke H, von Eckardstein A (1998) The emergence of triglycerides as a significant independent risk factor in coronary artery disease. Eur Heart J 19 [Suppl M]:M8-M14

7. Fontbonne A, Eschwege E, Cambien F et al. (1989) Hypertriglyceridaemia as a risk factor of coronary heart disease mortality in subjects with impaired glucose tolerance or diabetes. Results from the 11-year follow-up of the Paris Prospective Study. Diabetologia 32:300-304

8. Betteridge DJ (1999) Diabetic dyslipidaemia. Eur J Clin Invest 29 [Suppl 2]:12-16

9. Lamarche B, Despres JP, Moorjani S, Cantin B, Dagenais GR, Lupien PJ (1995) Prevalence of dyslipidemic phenotypes in ischemic heart disease (prospective results from the Quebec Cardiovascular Study). Am J Cardiol 75:1189-1195

10. Cui Y, Blumenthal RS, Flaws JA et al. (2001) Non-highdensity lipoprotein cholesterol level as a predictor of cardiovascular disease mortality. Arch Intern Med 161:14131419

11. Grundy SM (2001) Non-high-density lipoprotein cholesterol level as potential risk predictor and therapy target. Arch Intern Med 161:1379-1380

12. Frost PH, Havel RJ (1998) Rationale for use of non-highdensity lipoprotein cholesterol rather than low-density lipoprotein cholesterol as a tool for lipoprotein cholesterol screening and assessment of risk and therapy. Am J Cardiol $81: 26 \mathrm{~B}-31 \mathrm{~B}$ 
13. Havel RJ, Rapaport E (1995) Management of primary hyperlipidemia. N Engl J Med 332:1491-1498

14. Garg A, Grundy SM (1990) Management of dyslipidemia in NIDDM. Diabetes Care 13:153-169

15. Brewer HB Jr (1999) Hypertriglyceridemia: changes in the plasma lipoproteins associated with an increased risk of cardiovascular disease. Am J Cardiol 83:3F-12F

16. Mooy JM, Grootenhuis PA, Vries H de et al. (1995) Prevalence and determinants of glucose intolerance in a Dutch caucasian population. The Hoorn Study. Diabetes Care 18:1270-1273

17. Alberti KG, Zimmet PZ (1998) Definition, diagnosis and classification of diabetes mellitus and its complications. Part 1: diagnosis and classification of diabetes mellitus. Provisional report of a WHO consultation. Diabet Med 15:539-553

18. World Health Organization (1977) International classification of diseases, 9th rev. WHO, Geneva

19. Ballantyne CM, Olsson AG, Cook TJ, Mercuri MF, Pedersen TR, Kjekshus J (2001) Influence of low highdensity lipoprotein cholesterol and elevated triglyceride on coronary heart disease events and response to simvastatin therapy in 4S. Circulation 104:3046-3051

20. Despres JP, Lemieux I, Dagenais GR, Cantin B, Lamarche B (2000) HDL-cholesterol as a marker of coronary heart disease risk: the Quebec cardiovascular study. Atherosclerosis 153:263-272

21. Austin MA, Hokanson JE, Edwards KL (1998) Hypertriglyceridemia as a cardiovascular risk factor. Am J Cardiol 81:7B-12B

22. Criqui MH, Heiss G, Cohn R et al. (1993) Plasma triglyceride level and mortality from coronary heart disease. N Engl J Med 328:1220-1225

23. Patsch JR, Miesenbock G, Hopferwieser T et al. (1992) Relation of triglyceride metabolism and coronary artery disease. Studies in the postprandial state. Arterioscler Thromb 12:1336-1345

24. Gordon DJ, Probstfield JL, Garrison RJ et al. (1989) Highdensity lipoprotein cholesterol and cardiovascular disease. Four prospective American studies. Circulation 79:8-15
25. Gordon T, Castelli WP, Hjortland MC, Kannel WB, Dawber TR (1977) High density lipoprotein as a protective factor against coronary heart disease. The Framingham Study. Am J Med 62:707-714

26. Assmann G, Schulte H (1992) Role of triglycerides in coronary artery disease: lessons from the Prospective Cardiovascular Munster Study. Am J Cardiol 70:10H$13 \mathrm{H}$

27. Kannel WB, Castelli WP, Gordon T (1979) Cholesterol in the prediction of atherosclerotic disease. New perspectives based on the Framingham study. Ann Intern Med 90:8591

28. Bittner V, Hardison R, Kelsey SF, Weiner BH, Jacobs AK, Sopko G (2002) Non-high-density lipoprotein cholesterol levels predict five-year outcome in the Bypass Angioplasty Revascularization Investigation (BARI). Circulation 106:2537-2542

29. Frost PH, Davis BR, Burlando AJ et al. (1996) Serum lipids and incidence of coronary heart disease. Findings from the Systolic Hypertension in the Elderly Program (SHEP). Circulation 94:2381-2388

30. Lu W, Resnick HE, Jablonski KA et al. (2003) Non-HDL cholesterol as a predictor of cardiovascular disease in Type 2 diabetes: the Strong Heart Study. Diabetes Care 26:16-23

31. McGarry JD (2002) Banting lecture 2001: dysregulation of fatty acid metabolism in the etiology of type 2 diabetes. Diabetes 51:7-18

32. McNamara JR, Shah PK, Nakajima K et al. (2001) Remnant-like particle (RLP) cholesterol is an independent cardiovascular disease risk factor in women: results from the Framingham Heart Study. Atherosclerosis 154:229236

33. Heine RJ, Dekker JM (2002) Beyond postprandial hyperglycaemia: metabolic factors associated with cardiovascular disease. Diabetologia 45:461-475

34. De Vegt F, Dekker JM, Stehouwer CD, Nijpels G, Bouter LM, Heine RJ (2000) Similar 9-year mortality risks and reproducibility for the World Health Organization and American Diabetes Association glucose tolerance categories: the Hoorn Study. Diabetes Care 23:40-44 